\title{
A Short Note on Minimal Length
}

\author{
M. Moniruzzaman', S. B. Faruque ${ }^{2 *}$ \\ ${ }^{1}$ Department of Physics, Mawlana Bhashani Science and Technology University, Tangail 1902, \\ Bangladesh \\ ${ }^{2}$ Department of Physics, Shahjalal University of Science and Technology, Sylhet 3114, Bangladesh
}

Received 4 April 2018, accepted in final revised form 21 March 2019

\begin{abstract}
After revival of the concept of minimal length, many investigations have been devoted, in literature, to estimate upper bound on minimal length for systems like hydrogen atom, deuteron etc. We report here a possible origin of minimal length for atomic and nuclear systems which is connected with the fundamental interaction strength and the Compton wavelength. The formula we appear at is numerically close to the upperbounds found in literature.
\end{abstract}

Keywords: Minimal length; Generalized uncertainty principle; Bound systems.

(C) 2019 JSR Publications. ISSN: 2070-0237 (Print); 2070-0245 (Online). All rights reserved. doi: http://dx.doi.org/10.3329/jsr.v11i2.36244

J. Sci. Res. 11 (2), 151-155 (2019)

\section{Introduction}

The pursuit to know completely the nature of space-time in modern times dates back to the time of Einstein's and Heisenberg's epic works. Heisenberg first noticed that a nonzero minimal length is inevitable, but he could not incorporate it in a consistent theory [1]. But the history of a minimal length is becoming rich day by day. In the last decade of the 20th century, it became clear how a minimal length appears in quantum gravity [2], string theory [3], etc., and through the works of Kemf et al. [4-6], a deformed quantum mechanics appeared which incorporates a non-zero minimal length within a generalized uncertainty principle (GUP). The generalized uncertainty principle (GUP) is usually expressed as

$$
\Delta x \Delta p \geq \frac{\hbar}{2}\left[1+\beta(\Delta p)^{2}\right]
$$

where $\beta$ is a small parameter. Equation (1) leads to the minimal length $\Delta x_{\min }=\hbar \sqrt{\beta}$. Originally, this minimal length was envisaged as a gravitational induced uncertainty and

*Corresponding author: awsbf62@yahoo.com 
the scale of this length was usually understood to be of the order of Planck length $\left(10^{-35} \mathrm{~m}\right)$. But Eq.(1) contains the UV/IR mixing, whereby it is thought that the minimal length can be much larger than the Planck length.

Brau [7] calculated the effect of a minimal length on the spectrum of the hydrogen atom and estimated an upper bound on minimal length inherent in $\mathrm{H}$-atom which is of the order of $10^{-17} \mathrm{~m}$. Thereafter, many authors devoted time in estimating upper bound on minimal length inherent in many quantum systems and a large variety of results appeared. However, for hydrogen-like systems the estimated value of minimal length falls around the value found by Brau. We have previously estimated [8] the upper bound on minimal length using deuteron quadrupole moment which is about $10^{-16} \mathrm{~m}$.

Due to this development of the matter, some authors indicated that minimal length may depend on the systems under consideration. Moreover, Kemf [9] revealed that nonpointness of particles considerably affects energy spectrum of systems like harmonic oscillators, and Sastry [10] presented a theory of extended quantum particles where nonpointness of particles is shown to lead to non-zero minimal uncertainty in position. Influenced by these works, we are going to show here that a minimal length could be associated with every quantum system because of the non-pointness of the particles manifested by their being part of a system and by how their positions are measured. These two ingredients are inseparably associated with every quantum system and with the conceptual framework of Heisenberg uncertainty principle. And one obviously finds a minimal length to be operational in every quantum system when non-pointness of particles whose positions are measured is considered within the framework of quantum measurement. Through due consideration of non-pointness of particles, we arrive at a formula for minimal length. The work we present here is original and of fundamental importance for quantum physics. The paper is organized as follows: In Section 2, we derive the formula for minimal length and present some numerical values. In section 3 , we conclude.

\section{Minimal Uncertainty in Position}

According to Heisenberg's original thought experiment [11], a particle's position is measured through the interaction of a photon with the particle. The photon gets scattered and the scattered photon is viewed through a microscope which immediately leads to the ordinary uncertainty relation

$\Delta x \Delta p \geq \frac{\hbar}{2}$

Here, however, the particle, say electron, is considered as a point particle. But the interaction which is used to detect the electron always finds the particle as non-point like. This is manifest through the finite cross-section of the interaction which is always like Compton scattering. Every impingement reveals a finite size of the electron given by the low-energy limit of Compton scattering cross-section [12], 


$$
\sigma=\frac{8 \pi}{3}\left(\frac{e^{2}}{\hbar c} \frac{\hbar}{m_{e} c}\right)^{2}
$$

where $e$ is the charge of the electron and $m_{e}$ is the mass of electron. This is actually the Thomson scattering cross-section, the low energy limit of the Compton cross-section. So, a measure of non-pointness of the electron associated with Eq. (3) is given by a minimal length,

$$
\begin{aligned}
\Delta x_{\min } & \approx \frac{e^{2}}{\hbar c} \frac{\hbar}{m_{e} c} \\
& =\alpha \hbar_{c}
\end{aligned}
$$

where $\alpha$ is the fine structure constant and $\lambda_{c}$ is the reduced Compton wavelength of the electron.

Next, consider the H-atom. When energy of $\mathrm{H}$-atom is considered, the particle whose energy is measured is electron and its size is linked with the size of the orbit. In the ground state, the size of the electron, $\frac{\hbar}{m_{e} c}=\lambda_{c}$ and the radius of the orbit, Bohr radius $a_{0}$ are linked by

$$
\frac{\hbar_{c}}{a_{0}}=\alpha \simeq \frac{1}{137}
$$

If the electron is in a higher excited state the ratio $\frac{\lambda_{c}}{r_{n}}$ gradually approaches zero. Here lies another measure of non-pointness of the electron. This measure here is largest in the ground state. So, minimal size of the electron or it's non-pointness is not fixed if we consider the system where it is. The measure of this non-pointness varies between the minimum zero, which says it is really point like, and the maximum, $\alpha$ which says it is somewhat non-point like. But as long as we consider the electron in the $\mathrm{H}$ - atom this measure is never zero. For other particles in other systems, the measure of this nonpointness may turn out bigger. For example, in deuteron, the measure of non-pointness is more and can be taken to be $\alpha_{s} \approx 0.106$, where $\alpha_{s}$ is the coupling constant of strong interaction. We may find $\alpha_{s}$ by dividing the Compton wavelength of a nucleon by the size of deuteron (radious of deuteron, $r_{d}=1.964 \times 10^{-15} \mathrm{~m}$ ). This value is surprisingly very close to the value $\left(\alpha_{s} \approx 0.1\right)$ in quantum field theory [12].

Now, in literature, the systems for which the minimal length is being reported are bound systems like harmonic oscillator, hydrogen atom, deuteron, etc. Originally, the minimal length appeared for systems that are in Planck scale. For all such systems, the minimal length can be linked with the two pieces of non-pointness discussed above.

In case of the non-pointness associated with measurement through processes like Compton scattering, i.e., equation like Eq. (4), we have taken the low- energy limit because the particle we consider is bound and we wish to gather information by not 
disturbing the particle much. The Thomson cross section above corresponds to the case where the electron is not imparted large recoil energy. Moreover, a single scattering experiment with high incident photon energy would have sufficed to find the size of the particle in question, but the results of such experiment is not tractable much. That is why we propose the two pieces of non-pointness depicted above to be tied to find the minimal length. Now, for scattering experiment, the $\Delta x_{\min }$ is written as proportional to $\alpha_{I} \lambda_{c}$, where now $\alpha_{I}$ is the strength of interaction. For the non-pointness associated with the system where the particle is bound, i.e., equation like Eq. (5), we write $\Delta x_{\min } \propto \alpha_{B}$, where now $\alpha_{B}$ is the interaction strength corresponding to non-pointness due the system. We assume the minimal length to be proportional to both of those pieces. That is, $\Delta x_{\min } \propto \alpha_{I} \lambda_{c}$ and $\Delta x_{\min } \propto \alpha_{B}$. We thus write

$$
\Delta x_{\min } \sim \alpha_{I} \alpha_{B} \hbar_{c}
$$

For hydrogen and deuteron, we can take both of the interaction strengths to be the same. And for hydrogen, $\alpha \simeq \frac{1}{137}$ and $\lambda_{c}=\frac{\hbar_{c}}{m_{e} c}=3.86 \times 10^{-13} \mathrm{~m}$ and $\Delta x_{\min }$ turns to be about $2 \times 10^{-17} \mathrm{~m}$, which is close to Brau's value [7]. For deuteron, $\alpha_{s} \approx 0.1$ and $\lambda_{c} \simeq$ $\frac{\hbar}{m_{p} c}=2.1 \times 10^{-16} \mathrm{~m}$. Thus for deuteron, $\Delta x_{\min }$ turns out to be about $2.1 \times 10^{-18} \mathrm{~m}$, which is somewhat less than our previous value [8] and very close to the value that one can find using binding energy of deuteron [13]. For other systems, the two interaction strengths may not come out as the same and we have to compute the strengths considering the system and the experiment under question. For Planck scale system, $\lambda_{c} \approx 10^{-35} \mathrm{~m}$ and $\alpha_{p} \approx 1$, where $\alpha_{p}$ is the coupling constant for all the fundamental interactions at the Planck scale, and the minimal length turns out, as expected, to be the Planck length.

Thus, we arrive at a value for minimal length for bound systems as given by Eq. (6) and get some confirmation of the formula (6) from literature as quoted above. Strictly speaking, formula (6) represents the upperbound on minimal length for bound systems and can be linked with the uncertainties of dynamical properties of such systems. However, a minimal length for every quantum system is inevitable which is clear from the discussion above and this minimal length can be fairly considered to be not more than that given by Eq. (6). For systems bound by fundamental interaction, Eq. (6) is easy to evaluate, but for other systems, further investigation is necessary.

\section{Conclusion}

Minimal length first appeared in string theory, quantum gravity, and related fields is the minimal uncertainty in position measurement of a quantum particle. In this paper, we have argued that a minimal length is inevitable in Heisenberg's uncertainty principle. The long silence about minimal length after Heisenberg's original formulation of the uncertainty principle, is due to its very small size. The most fundamental minimal length is of the Planck scale, but in bound systems like hydrogen atom, deuteron etc., the minimal length 
can appear to be much bigger. A minimal length can be considered to be operational in every quantum system, which is shown in this work to be linked with non-pointness of particles like electron, nucleon, etc. The non-pointness discussed in this paper has two pieces. One is linked with the particles being part of a bound system and the other is linked with the method of measurement of the particle's position. The measure of nonpointness, when we consider a particle within a quantum system, is given approximately by the coupling strength of the interaction by which the particle is bound within the system. The other measure of non-pointness, when we consider the way of position measurement, is given approximately by the square root of interaction cross section, the interaction being like Compton scattering. We thus arrived at the formula (6) for minimal length whose numerical value is close to the upperbounds on minimal length found in literature. Therefore, we find a significant piece of physics in this work. Further investigation on this particular origin of minimal length in quantum systems may shed more light on the topic.

\section{References}

1. B. Carazza and H. Kragh, Am. J. Phys. 63, 595 (1995). https://doi.org/10.1119/1.17848

2. L. J. Garay, Int. J. Modern Phys. A10, 145 (1995). https://doi.org/10.1142/S0217751X95000085

3. K. Konoshi, G. Paffuti, and P. Provero, Phys. Lett. B234, 276 (1990). https://doi.org/10.1016/0370-2693(90)91927-4

4. A. Kempf, G. Mangano, and R. B. Mann, Physical Rev. D 52, 1108 (1995). https://doi.org/10.1103/PhysRevD.52.1108

5. A. Kempf and G. Mangano, Physics Rev. D55, 7909 (1997). https://doi.org/10.1103/PhysRevD.55.7909

6. A. Kempf, On the Structure of Space -Time at the Planck Scale (1998). https://arxiv.org/pdf/hep-th/9810215.pdf

7. F. Brau, J. Phys. A: Mathemat. General 32, 7691 (1999). https://doi.org/10.1088/03054470/32/44/308

8. S. B. Faruque, A. Rahman, and M. Moniruzzam, Result in Physics 4, 52 (2014). https://doi.org/10.1016/j.rinp.2014.05.002

9. A. Kempf, J. Phys. A: Mathemat. General 30, 2093 (1997). https://doi.org/10.1088/03054470/30/6/030

10. R. R. Sastry, J. Phys. A: Mathemat. General 33, 8305 (2000). https://doi.org/10.1088/03054470/33/46/311

11. W. Heisenberg, The Physical Principles of the Quantum Theory (Dover Publications, USA, 1930).

12. F. Mandl and G. Show, Quantum Field Theory, $2^{\text {nd }}$ Edition (John Wiley \& Sons, UK, 2010).

13. M. Moniruzzaman and S. B. Faruque, J. Sci. Res. 10, 99 (2018). https://doi.org/10.3329/jsr.v10i2.32829 